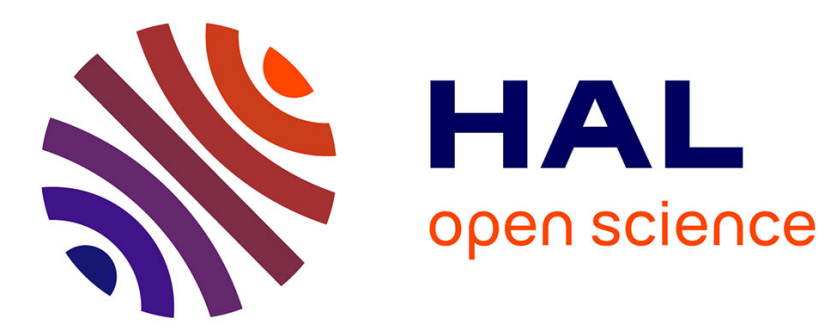

\title{
Rapport du préfet de police de Paris sur les cabales dans les théâtres parisiens (27 juin 1822)
}

Sylvain Nicolle

\section{To cite this version:}

Sylvain Nicolle. Rapport du préfet de police de Paris sur les cabales dans les théâtres parisiens (27 juin 1822). Parlement $[\mathrm{s}]$, Revue d'histoire politique, 2012, nºHS8 (3), pp.169. 10.3917/parl.hs08.0169 . hal-03029927

\section{HAL Id: hal-03029927 https://hal.science/hal-03029927}

Submitted on 29 Nov 2020

HAL is a multi-disciplinary open access archive for the deposit and dissemination of scientific research documents, whether they are published or not. The documents may come from teaching and research institutions in France or abroad, or from public or private research centers.
L'archive ouverte pluridisciplinaire HAL, est destinée au dépôt et à la diffusion de documents scientifiques de niveau recherche, publiés ou non, émanant des établissements d'enseignement et de recherche français ou étrangers, des laboratoires publics ou privés. 


\section{Rapport du préfet de police de Paris sur les cabales dans les théâtres parisiens (27 juin 1822)}

\section{Sylvain Nicolle}

Doctorant à l'Université de Versailles Saint-Quentin-en-Yvelines, CHCSC

sylvain.nic1@voila.fr.

"Avec, et peut-être avant les journaux, le plus puissant moyen, au XIX siècle, pour émouvoir l'opinion, c'est le théatre »". Difficile de trouver formulation plus éloquente - et qui n'a rien d'exagéré - pour prendre la mesure de la place occupée par le théatre dans la société française du premier XIX' siècle, et de la Restauration en particulier.

1 Archives Nationales, F21 966. Procès-verbal de censure de Laurent de Médicis, daté de février 1824 mais non signé.

${ }^{2}$ NDLR : voir aussi sur ce sujet, supra, dans la rubrique [Recherches], Corinne Legoy, "Sous la plume du pouvoir, le public de théâtre entre 1815 et 1830 : l'embarrassant miroir d'une nation souveraine ", p. 95-108, et Jean-Claude Yon, "Le Fils de Giboyer (1862) d'Émile Augier: un scandale politique au théâtre sous le Second Empire », p. 109-122. 
[Sources]

Le document reproduit ci-dessous est un rapport du préfet de police, adressé à la fois au ministre de l'Intérieur (Corbière) et au ministre de la Maison du Roi (Lauriston), et daté du 27 juin 18223. Son auteur, Delavau, a été nommé à la préfecture de police en décembre 1821, quelques jours après la formation du ministère Villèle qui permet aux Ultras de retrouver l'exercice du pouvoir. Dans ce rapport, le préfet tente de politiser la cabale, dont la manifestation dans les théatres déciderait en toute impunité du verdict des pièces représentées pour faire triompher l'opposition libérale, selon lui. Le vocabulaire employé, très fluctuant, est au service de l'objectif du préfet: "bande de cabaleurs 》, " cabale 》 et "race de claqueurs de commande 》 sont considérés comme synonymes. À l'évidence, le préfet confond volontairement la cabale (opposition ou soutien bruyants qui peuvent venir de n'importe quel groupe de spectateurs) et la claque (les spectateurs généralement payés pour applaudir ou siffler), ce qui permet d'atténuer la portée politique des troubles en rejetant leur origine sur les seuls claqueurs, véritables professionnels du "désordre établi》 dans les théâtres. Or si tous les claqueurs sont par définition des cabaleurs, la réciproque ne se vérifie pas toujours: une note sur les claqueurs émanant de la Maison du Roi et que l'on peut dater de 1825 atteste que Henry, chef de claque de l'Odéon, est régulièrement la cible des attaques des étudiants qui occupent le parterre de l'Odéon ${ }^{4}$. Ainsi, ce rapport est-il à la fois un lamento littéraire sur la décadence de l'art dramatique provoquée par la claque et un plaidoyer politique qui s'inquiète de la contestation orchestrée par les Libéraux au théâtre pour faire de celui-ci une seconde tribune. N'est-il pas précisément rédigé à un moment où l'opposition libérale, très amoindrie par ses défaites électorales, peine à faire entendre sa voix à la tribune de la Chambre?

"J'ai l'honneur d'appeler l'attention de Votre Excellence sur les cabales des théâtres.

Ces cabales qui dans les dernières années ont été souvent l'objet de la surveillance particulière de l'autorité, sont organisées de telle sorte qu'elles devraient finir par corrompre entièrement le goût d'un parterre qui serait encore éclairé ; car elles applaudissent à outrance ou sifflent avec fureur, suivant que leur intérêt les dirige, sans être capables d'examiner si les sujets applaudis ou sifflés ne mériteraient pas un succès tout contraire. Ces bandes de cabaleurs marchent sous des chefs connus dans chaque théâtre. Les auteurs et les acteurs sont obligés de leur payer tribut, soit en argent, soit en billets, et ce qu'il y a de plus

3 A.N, cartons $\mathrm{F}^{21} 1045$ et $\mathrm{O}^{3} 1620$.

${ }^{4}$ Nous avons consacré un chapitre sur les liens complexes entre cabale et claque dans un travail auquel nous nous permettons de renvoyer: Sylvain Nicolle, Les Théatres parisiens et le pouvoir sous la Restauration : quelle politique théâtrale? (1814-1830), mémoire de Master 2 sous la direction de Jean-Yves Mollier et Jean-Claude Yon, Université de Versailles-Saint-Quentin, 2010, p. 238-265. L'auteur prépare une thèse intitulée Les débats parlementaires sur le théâtre en France au XIX siècle (1820-1914). 
scandaleux, c'est que le plus souvent un auteur, après avoir dépensé d'avance une partie du revenu qu'il espérait de ses travaux littéraires, se trouve subitement abandonné par la cabale qui devait le servir, et qui aide à sa défaite après avoir partagé ses dépouilles. C'est ainsi que dernièrement, M. Mély-Janin, auteur d'Oreste, s'est vu sifflé par la même cabale qu'il avait payée pour en être applaudi ${ }^{5}$. Ce scandale vient de l'opinion naturellement libérale des individus qui font un si vil trafic. Ils reçoivent de l'argent des mains royalistes, mais les vers royalistes n'en sont pas moins sacrifiés à leur opinion particulière. Voilà pourquoi, depuis plusieurs années, on voit un si grand nombre de succès libéraux ${ }^{6}$, quoiqu'il soit bien certain que le libéralisme n'a produit encore que des déclamations tragiques et des senteurs dix fois usées depuis celles qui fourmillent dans les pièces de Voltaire.

Quoi qu'il en soit, je pense que les cabales sont évidemment contraires à l'intérêt de l'art et à l'ordre public; qu'un gouvernement fort ne peut pas tolérer ces scandales; enfin que si l'on veut ramener la paix dans les théâtres et laisser en même temps, au parterre toute indépendance de ses jugements, il faut se hâter d'en bannir ces troupes de désœuvrés ignorants et serviles qui ne savent que trépigner aux mots de liberté, et qui sont inflexibles aux beautés de la poésie.

Déjà, dans les années 1809, 1811 et 1812, la police a cherché à détruire cette race de claqueurs de commande ${ }^{7}$. Alors plus maitresse de ses actes qu'elle ne peut l'être aujourd'hui, elle prit le parti nécessaire de faire disparaittre les chefs de cabales en leur faisant subir un emprisonnement qui, à l'égard de quelques uns eut même lieu à plusieurs reprises, suivant qu'ils avaient été plus ou moins hardis à braver les défenses de l'autorité. Aujourd'hui si les actes arbitraires ne peuvent être mis en usage, même dans le sens dont il s'agit, il semble, Monseigneur, que l'autorité peut du moins donner aux administrations ou directeurs des théâtres l'ordre de fermer leurs établissements aux cabaleurs, en fixant à un nombre très limité les billets de faveur accordés par les auteurs pour les jours de première représentation de leurs ouvrages, et par les acteurs qui paraissent pour la première fois sur

\footnotetext{
5 Oreste, tragédie en 5 actes de Mély-Janin représentée à l'Odéon le 16 juin 1821. Voir le récit fiable qu'en donne Théodore Muret dans L'Histoire par le théâtre, 1789-1851, t. 2, Paris, 1865, p. 202-203. La pièce participe de ce qu'il appelle « les pièces immolées sur l'autel de la politique $»$.

${ }^{6}$ On peut penser en particulier à Germanicus d'Arnault (1817), Les Vêpres Siciliennes de Delavigne (1820), Sylla de Jouy (1821), Régulus d'Arnault fils (1822).

${ }^{7}$ Voir le rapport de la préfecture de police intitulé Théatres : cabaleurs, $n^{\circ} 1$. Travail général sur les chefs de cabale 1809, 1811, 1812. Une copie est reproduite dans Louis Allard, La Comédie de mours en France au XIX siècle (1795-1830), t. 1, Paris, Hachette, 1923, p. 485-490.
} 
la scène. Le théâtre de la Porte Saint-Martin a fourni naguère la preuve qu'une telle mesure est praticable et qu'elle peut très bien réussir. Ce théâtre en effet s'est délivré de la cabale, et depuis lors, tout se passe parfaitement dans son parterre ${ }^{8}$. Plus récemment encore le Vaudeville a imité cet exemple, et tout fait penser que cette mesure aura le même succès à ce théâtre.

Si donc l'administration supérieure, d'après les différentes considérations que je viens de mettre sous ses yeux, voulait bien adopter mon opinion, et juger convenable que la mesure prise par les théâtres de la Porte Saint-Martin et du Vaudeville, fût de même appliquée aux autres thêâtres, je ne puis assez répéter qu'elle ferait une chose éminemment utile dans l'intérêt de l'ordre public, et j'ajoute même dans l'intérêt de l'art dramatique. Il n'est pas dans ma pensée de développer les considérations qui s'offriraient d'elles-mêmes, à n'envisager la chose que sous le rapport du goût. Mais je dois rappeler à Votre Excellence quelques-uns des succès récents acquis en grande partie par la cabale à des tragédies républicaines, et si l'on vient à comparer le mérite de ces pièces avec la vogue qu'elles obtiennent encore, on est contraint de se demander si l'art n'est pas menacé de décadence, lorsqu'il suffit de quelques tirades révolutionnaires pour exciter l'enthousiasme, et que les parterres sont envahis par des juges, qui au lieu d'applaudir au talent littéraire ne savent qu'encourager les passions politiques.

En provoquant ainsi la décision qui pourrait intervenir sur l'objet de la présente, j'ai l'honneur de prier Votre Excellence de vouloir bien me donner connaissance des suites dont mes propositions lui auront paru susceptibles. »

\footnotetext{
${ }^{8}$ Depuis le mois de mai 1822, le Théâtre de la Porte Saint-Martin avait procédé à une réforme en deux volets: $1^{\circ}$ suppression des billets de faveur donnant accès au parterre ; $2^{\circ}$ Conversion des billets de service donnés aux acteurs en carte d'entrée nominative. En cas de fraude, la carte d'entrée confiée à un claqueur devait être immédiatement détruite et le comédien en était privé définitivement. La presse salue cette initiative mais cette mesure fut inefficace pour deux raisons : d'une part, elle ne concernait pas les auteurs, d'autre part, son caractère fut très éphémère : dès la fin du mois de juillet, les claqueurs étaient réintroduits en plus grand nombre. Ce dernier fait sert de prétexte à la Maison du Roi dans sa réponse au préfet pour refuser une véritable réforme des billets de faveur dans les théâtres royaux.
} 